\title{
Developmental Expression Profile of the Optic Atrophy Gene Product: OPA1 Is Not Localized Exclusively in the Mammalian Retinal Ganglion Cell Layer
}

\author{
Saima Aijaz, ${ }^{1}$ Lynda Erskine, ${ }^{1,2}$ Glen Jeffery, ${ }^{2}$ Shomi S. Bbattacharya, ${ }^{1}$ and \\ Marcela Votruba ${ }^{1,3}$
}

\begin{abstract}
Purpose. Autosomal dominant optic atrophy (ADOA) is characterized by primary degeneration of retinal ganglion cells and atrophy of the optic nerve. The OPA1 gene encodes a 960amino-acid protein. In the current study the temporal and spatial localization of OPA1 were examined in developing and adult murine ocular tissues and the adult human eye. Because the $B s t /+$ mouse has been postulated as a model of ADOA, the mOPA1 expression in the Bst/+ retina was also examined.
\end{abstract}

METHODs. A polyclonal antibody generated against a C-terminal peptide of OPA1 was used to assess by immunohistochemistry the expression of mOPA1 in the wild-type embryonic and postnatal mouse ocular tissues and the Bst/+ retina. Western blot analyses of total proteins from a panel of adult human tissues were used to examine the expression of human OPA1, and spatial localization was assessed by immunohistochemistry.

Results. The ocular expression of mOPA1 begins at E15 in the inner retina in a location corresponding to that of the subsequently developing ganglion cell layer (GCL) and peaks between postnatal day $(\mathrm{P}) 0$ and $\mathrm{P} 1$ in the retina and the optic nerve. There is a sharp decline in mOPA1 expression after P2, but it is expressed at a basal level until at least P12 in the GCL, inner plexiform layer (IPL), and inner nuclear layer (INL) of the retina as well as in the optic nerve. In the adult Bst/+ retina, mOPA1 is strongly expressed in the GCL and IPL and weakly in the INL. In the adult human eye, OPA1 is expressed in the GCL, IPL, INL, and outer plexiform layer (OPL) of the retina and in the optic nerve, where it is observed only in the myelinated region.

Conclusions. OPA1 is not restricted to the GCL of the mammalian retina, and its expression extends into the IPL, INL, and OPL. OPA1 is distinctly expressed in the myelinated region beyond the lamina cribrosa in the human optic nerve, whereas its expression is weaker in the mouse optic nerve. In the Bst/+ mouse retina, despite the structural defects, mOPA1 expres-

From the Departments of ${ }^{1}$ Molecular Genetics and ${ }^{2}$ Visual Science, Institute of Ophthalmology, London, United Kingdom; and ${ }^{3}$ Moorfields Eye Hospital, London, United Kingdom.

Presented in part at the annual meeting of the Association for Research in Vision and Ophthalmology, Fort Lauderdale, Florida, May 2003.

Supported by the Wellcome Trust and the Birth Defects Foundation, United Kingdom.

Submitted for publication October 2, 2003; revised December 10, 2003, and January 8, 2004; accepted January 16, 2004.

Disclosure: S. Aijaz, None; L. Erskine, None; G. Jeffery, None; S.S. Bhattacharya, None; M. Votruba, None

The publication costs of this article were defrayed in part by page charge payment. This article must therefore be marked "advertisement" in accordance with 18 U.S.C. $\$ 1734$ solely to indicate this fact.

Corresponding author: Marcela Votruba, Cell and Molecular Biology Group, Cardiff School of Optometry and Vision Sciences, Cardiff University, Redwood Building, King Edward VII Avenue, Cardiff CF10 3ND, UK; m_votruba@yahoo.co.uk. sion is comparable to that observed in the wild-type adult mouse retina. These observations suggest a wider role for OPA1 than previously anticipated. (Invest Ophthalmol Vis Sci. 2004;45:1667-1673) DOI:10.1167/iovs.03-1093

A utosomal dominant optic atrophy (ADOA) is the most A prevalent primary hereditary optic neuropathy, with a frequency of $1: 12,000$ to $1: 50,000$ in developed countries. ${ }^{1}$ ADOA is frequently diagnosed by 6 to 8 years of age, but cases with nystagmus and onset as early as 1 year of age are well recognized, confirming the congenital nature of the disease in some patients. ${ }^{2}$ The disease is characterized by an insidious onset of visual impairment in early childhood with moderate to severe loss of visual acuity, temporal optic disc pallor, color vision deficits, and centrocecal scotoma of variable density. ${ }^{3-5}$ ADOA shows variable expression ranging from asymptomatic carriers to legally blind patients ${ }^{4}$ and is untreatable by surgical or medical interventions. Evidence from electrophysiological and histopathological studies shows that the fundamental underlying defect is retinal ganglion cell (RGC) degeneration followed by ascending atrophy of the optic nerve, including loss of myelin and nerve tissue within the optic nerve. ${ }^{3,6-8}$

There are four loci for nonsyndromic optic atrophy, ${ }^{9-12}$ but the most common is $O P A 1$, which is attributable to mutations in the OPA1 gene located at q28 on chromosome $3 .{ }^{13-16}$ The OPA1 gene spans 70 to $90 \mathrm{~kb}$ and is composed of 31 exons. ${ }^{17}$ Northern blot analyses of OPA1 transcripts have shown the presence of OPA1 RNA in almost all human tissues examined. ${ }^{13,16}$ The last exon is not coded, and exons $4,4 \mathrm{~b}$, and $5 \mathrm{~b}$ generate eight isoforms of RNA by alternative splicing, with some splicing isoforms predominating in the brain, retina, heart, and muscle. ${ }^{17}$

OPA1 encodes a protein of 960 amino acids, which shows homology to yeast mitochondrial dynamin-like guanosine triphosphate (GTP) binding proteins, due to the presence of an $\mathrm{N}$-terminal leader sequence for mitochondrial localization and central guanosine triphosphatase (GTPase) and dynamin domains. ${ }^{13,16}$ The $\mathrm{C}$ terminus of OPA1 differs from other dynamin family members in lacking a proline-rich region as well as dynamin GTPase effector and pleckstrin homology domains. ${ }^{18}$ Primary structure predictions suggest a high probability of coiled-coil formations in two separate domains encoded by exons $5 \mathrm{~b}$ to 7 (90\%-100\%) and exons 27 to 28 (95\%), and these may be involved in the formation of homodimers or polymers or the binding of as yet unknown cellular partners. Studies in yeast have demonstrated that native GTPases of the Mgm1/ Msp1 subfamily, which show the highest homology to OPA1, play an important role in the dynamics of mitochondrial morphology and the fusion/fission processes. ${ }^{19-22}$ Different isoforms of OPA1 have been sublocalized to the inner and outer mitochondrial membranes. ${ }^{23,24}$

Several hypotheses have been put forward to explain the pathogenic mechanisms that may play a role in OPA1-type dominant optic atrophy. Truncating mutations in the N-terminal region and missense mutations in the GTPase domain lead 
to loss of function of the protein and haploinsufficiency. ${ }^{17,25-27}$ Other mutations that result in the loss of the last seven C-terminal amino acids or skipping of exon 27 suggest that the last two exons encode a critical domain in OPA1 structure or function. ${ }^{25-28}$ Because exons 27 to 28 have a high probability of forming a coiled-coil domain, which putatively interacts with other proteins, these truncated OPA1 proteins may be impaired in their interaction with other proteins and exert a dominant negative effect. Although most missense and truncating mutations have been found predominantly in the GTPase or C-terminal regions, the pathologic effect is observed only in the RGCs. ${ }^{3}$ This is intriguing, given the fact that OPA1 transcripts are ubiquitously expressed in all body tissues. ${ }^{13,16}$ Previous reports have speculated that this may be due to OPA1 being predominantly expressed in the $\mathrm{RGCs}^{26}$ or the RGCs' being more susceptible to reactive oxygen species. ${ }^{29}$ To date, there are no data to suggest a role for OPA1 in the degeneration of the RGCs.

Patients with ADOA have a reduction in the number of RGCs. ${ }^{3,6}$ In 1977, Southard and Eicher ${ }^{30}$ identified an autosomal, semidominant mutation, belly spot and tail (Bst), which arose spontaneously in the C57BLKS mouse strain. ${ }^{30}$ Whereas mice homozygous for Bst die in utero, adult heterozygotes are viable and fertile and show reduced or complete absence of the pupillary light reflex in one or both eyes and unilateral or bilateral atrophy of the optic nerve. ${ }^{31}$ This is similar to human ADOA, in which patients show variable expression ranging from asymptomatic carriers to complete blindness. As a result, the $B s t /+$ mouse has been proposed as a mouse model of optic atrophy. ${ }^{32}$ However, Bst/ + mice also show defects such as polydactyly and retinal coloboma and rosette formation in addition to a decrease in the number of RGCs. ${ }^{31,33,34}$ These studies suggested that the Bst gene may play a developmental role in regulating cell proliferation during organogenesis.

To understand the functional role of OPA1 in normal retinal development and to shed light on the pathogenesis of ADOA, we examined the expression pattern of mOPA1 in the prenatal and postnatal eyes of mouse and compared the murine expression profile with the expression of OPA1 in human ocular tissues. We also examined the expression of mOPA1 in the $B s t /+$ retina compared with that in the wild-type retina.

\section{Methods}

\section{Preparation of Tissues}

All protocols involving the use of mice adhered to the regulations set forth in the ARVO Statement for the Use of Animals in Ophthalmic and Vision Research. Experiments involving human tissue conformed to the guidelines set forth in the Declaration of Helsinki for the use of human tissue in research, and we had prior approval from the Moorfields Eye Hospital Research and Ethics Committee.

Mouse Embryos. Wild-type mouse embryos were obtained from mating of male and female BALB/c mice. Mating was assumed to have taken place at midnight, and the day of plug detection until the following midnight was called embryonic day (E)0. Embryos were dissected from pregnant females at E14, E15, E16, and E17 and fixed in $4 \%$ paraformaldehyde in phosphate-buffered saline (PBS) overnight at $4^{\circ} \mathrm{C}$. Subsequently, the embryos were dehydrated and embedded in paraffin wax for sectioning at 5 to $6 \mu \mathrm{m}$. Two pregnant females were used at each time point, and data were compiled from results taken from experiments on at least three embryos from each time point.

Postnatal Mouse Eyes. Timed-pregnant C57BL/6J mice were purchased from Harlan (Crawley Down, UK) The day of plug detection was designated as E0. Eyes were dissected rapidly from newborn mice starting from the day of birth (designated as PO or postnatal day [P]0) to P12 and immediately transferred to freshly prepared $4 \%$ paraformaldehyde in PBS for overnight fixation at $4^{\circ} \mathrm{C}$. After fixation, the eyes were dehydrated and embedded in paraffin wax. Sections were cut at $6 \mu \mathrm{m}$ for use in immunohistochemistry. Data were compiled from results taken from experiments on eyes from two different mice at each time point. Adult mouse eyes were obtained from C57BL/6J mice, fixed in $4 \%$ paraformaldehyde in PBS overnight, dehydrated, and embedded in paraffin wax. Sections were cut at $6 \mu \mathrm{m}$ for use in immunohistochemistry.

Bst Mouse Eyes. Eyes from 10-week-old Bst/+ mice on C57BL/6J background were obtained from the Jackson Laboratory (Bar Harbor, ME) in $10 \%$ neutral buffered formalin. The eyes were dehydrated, equilibrated with xylene, and embedded in paraffin wax. Sections were cut at $6 \mu \mathrm{m}$ for use in immunohistochemistry.

Human Eye Tissue. A donor eye from a 68-year-old patient was obtained from the Moorfields Hospital Eye Bank with full prior approval from the Moorfields Eye Hospital Research and Ethics Committee and fixed in $10 \%$ neutral-buffered formalin, within 2 hours of enucleation, for 24 hours. The eye was dehydrated with increasing concentrations of industrial methylated spirit (IMS), equilibrated in xylene, and embedded in paraffin wax. Sections were cut at $6 \mu \mathrm{m}$ on a microtome, floated on $20 \%$ methanol, expanded on water preheated to $40^{\circ} \mathrm{C}$, and mounted on glass slides.

\section{Antibody Generation, SDS-PAGE, and Immunoblot Analysis}

A peptide corresponding to amino acids $938-960$ of the human OPA1 sequence (DLKKVREIQEKLDAFIEALHQEK) was synthesized and conjugated to keyhole limpet hemocyanin (KLH) through an artificial Cys placed at the N-terminal (Genosys Biotechnologies, Cambridge, UK). Rabbit polyclonal antiserum (anti-OPA1) was obtained by subcutaneous injection of the conjugated peptide every 4 weeks (Genosys Biotechnologies).

Total protein extracts from different human tissues were prepared by homogenization in sample buffer $(0.0625 \mathrm{M}$ Tris-HCl, $2.5 \%$ SDS, 5\% 2-mercaptoethanol, $10 \%$ glycerol, and $0.25 \%$ bromophenol blue). Total protein concentrations in tissue extracts were assayed using the Bradford microassay (Bio-Rad Laboratories, Hercules, CA) to ensure even loading of gels. Protein samples from each extract were loaded on $12 \%$ SDS-PAGE gels and resolved by electrophoresis. The gels were electroblotted onto ECL nitrocellulose (Amersham, UK). The nitrocellulose membrane was incubated overnight in blocking solution ( $5 \%$ nonfat milk powder, $1 \times$ PBS, and $0.1 \%$ Tween-20), after which the blots were incubated with either rabbit preimmune serum or anti-OPA1 (1:2000 dilution) antibody for 1 hour at room temperature (RT). In competition assays, the peptide $(1 \mu \mathrm{g} / \mathrm{mL})$ against which the antiserum was generated was incubated with the anti-OPA1 antiserum (1:2000) for 30 minutes on ice before incubation with the nitrocellulose membrane for 1 hour at RT. Subsequently, the membrane was incubated with goat anti-rabbit secondary antibody (1:30,000) conjugated to horseradish peroxidase (Jackson ImmunoResearch Laboratories, West Grove, PA) for 1 hour at RT. Bands were visualized by enhanced chemiluminescence using the ECL kit (Amersham).

\section{Immunohistochemistry}

Paraffin-embedded sections of mouse embryos, postnatal mouse eyes, $B s t /+$ mouse eyes, and human eye were deparaffinated with xylene and rehydrated successively with $100 \%, 95 \%$, and $70 \%$ IMS. Endogenous peroxidase activity was blocked by incubating the sections with $0.5 \%$ hydrogen peroxide (Sigma-Aldrich, Poole, UK) in 50\% methanol for 30 minutes at RT. The sections were then blocked with $10 \%$ normal goat serum (Dako, Ltd., High Wycombe, UK) in Tris-buffered saline (TBS) for 20 minutes at RT, followed by incubation with anti-OPA1 antibody (1:100 or 1:250 dilution) in TBS containing 10\% normal goat serum overnight at $4^{\circ} \mathrm{C}$. Subsequently, the sections were briefly rinsed with TBS and incubated in goat anti-rabbit antibody conjugated to horseradish peroxidase (1:500; Jackson ImmunoResearch Laboratories) for 45 minutes at RT. The immunoreaction was visualized by staining with solution containing $3^{\prime}, 3^{\prime}$-diaminobenzidine (DAB; Sigma-Aldrich), 

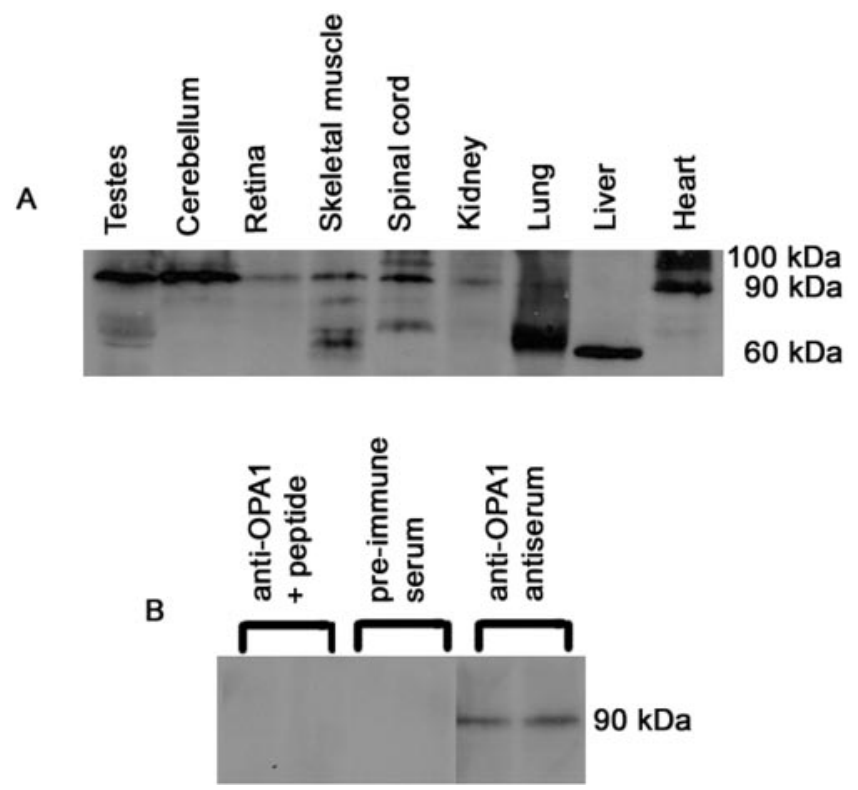

FigURE 1. (A) Immunoblot of proteins from a panel of human tissues, using the anti-OPA1 antiserum. The antiserum detected proteins of $\sim 90 \mathrm{kDa}$ in most tissues and a larger band of $\sim 100 \mathrm{kDa}$ in the heart and spinal cord. A smaller band of $\sim 60 \mathrm{kDa}$ was also detected in the liver and skeletal muscle. (B) Immunoblot of human retinal proteins reacted with anti-OPA1 antiserum. A single band of $\sim 90 \mathrm{kDa}$ was detected in the human retina by anti-OPA1 antiserum. Rabbit preimmune serum did not detect the $\sim 90-\mathrm{kDa}$ band, and the band was not detected when the anti-OPA1 antiserum had been preadsorbed by the peptide against which it was generated before immunoblot analysis.

$0.03 \%$ hydrogen peroxide in TBS until color development. Some sections were developed with alkaline phosphatase substrate (Vector Red; Vector Laboratories Inc., Burlingame, CA). Subsequently, the sections were successively dehydrated with 70\%, 95\%, and 100\% IMS, clarified with three changes of xylene, and mounted in dibutyl phthalate xylene (DPX) mounting medium (Merck, Hoddesdon, UK). To verify the specificity of the immunostaining, sections were also stained with rabbit preimmune serum. The sections were visualized with a light microscope (BX50; Olympus, Tokyo, Japan) using bright-field and differential interference contrast (Nomarski) optics and photographed with a digital camera (model DXM1200; Nikon, Tokyo, Japan).

\section{Results}

\section{Characterization of OPA1 Expression in Human Tissues}

A rabbit polyclonal antibody was raised against a KLH-conjugated peptide corresponding to amino acids 938 to 960 of the predicted human OPA1 sequence. The specificity of this antibody (anti-OPA1) was confirmed by Western blot analysis of total protein extracts from nine different human adult tissues including the cerebellum, spinal cord, retina, heart, liver, and lung.

A major band of approximately $90 \mathrm{kDa}$ was detected in total protein extracts from the human heart, lung, kidney, spinal cord, skeletal muscle, retina, cerebellum, and testes under reducing conditions (Fig. 1A). No protein was detected when the total protein extract from the retina was incubated with the anti-OPA1 antibody in the presence of the competing peptide or by incubation with rabbit preimmune serum, indicating that the reactivity against the $90 \mathrm{kDa}$ band was specific (Fig. 1B). A larger band of approximately $100 \mathrm{kDa}$ was also detected in the heart and spinal cord (Fig. 1A). This is consistent with a previous report that showed that OPA1 exhibits eight RNA isoforms as a result of alternative splicing of exons $4,4 \mathrm{~b}$, and $5 \mathrm{~b}$ and that some isoforms predominate in different tissues. ${ }^{17}$ The mature OPA1 protein has a predicted molecular weight of approximately $106 \mathrm{kDa}$, and this may account for the $100 \mathrm{kDa}$ band that we detected in total protein extracts from the heart and spinal cord. We also detected a $60 \mathrm{kDa}$ band in the liver. The identity of this smaller band is unclear, because it is too small to be a known isoform of OPA1. Smaller OPA1 RNA transcripts of approximately 4 and $3.5 \mathrm{~kb}$ have been reported in the human and mouse livers, respectively, by Northern analyses and have been attributed to alternative polyadenylation. ${ }^{13,16,20}$ In another study, the smaller $86 \mathrm{kDa}$ protein band observed in the mitochondrial fraction of rat liver using a different anti-OPA1 antibody was attributed to posttranslational modifications and/or aberrant conformational migration. ${ }^{23}$ Whether the $60 \mathrm{kDa}$ protein currently of uncertain identity detected by our anti-OPA1 antibody in the liver is a result of alternative polyadenylation, or post translational modification, or both needs to be examined further. Also, it is unclear why only this $60 \mathrm{kDa}$ band is detected in the liver.

\section{Expression of mOPA1 in the Developing Mouse Embryo}

Because the underlying pathology in OPA1-type optic atrophy is primary degeneration of $\mathrm{RGCs},{ }^{3}$ we investigated the temporal and spatial expression profile of mOPA1 in the developing mouse retina from embryonic stages E14 to E17. We did not see any ocular expression of mOPA1 in the E14 embryo (data not shown). This may either reflect the absence of mOPA1 or lack of sensitivity of immunohistochemical techniques in detecting low levels of protein. At E15, mOPA1 begins to appear in the prospective ganglion cell layer (GCL) of the developing retina (Fig. 2B). At the E16 stage, strong expression of mOPA1 was observed in the prospective GCL as well as in the lens and the ectoderm overlying the eye (Fig. 2C) and the brain (data not shown). A low level of expression was also observed in the neuroblastic layer (Fig. 2Cc). No signal was detected when the anti-OPA1 antibody was omitted or when the sections were stained only with rabbit preimmune serum (Fig. 2A), confirming the specificity of the mOPA1 signal. Just before birth, at E17, mOPA1 was very strongly expressed only in the GCL of the retina. No expression was observed in the retinal pigmented epithelium (Fig. 2D) or other ocular tissues. However, strong mOPA1 expression was detected in most other body tissues of the embryo, including the surface ectoderm, somites, heart, liver, lung, brain (data not shown), and skeletal muscle (Fig. 2E).

\section{Postnatal Expression Profile of mOPA1 in Mouse Ocular Tissues}

Strong expression of mOPA1 was observed in the GCL of the retina at $\mathrm{P0}$ (Fig. 3A) and continued until the $\mathrm{P} 2$ stage, peaking at P1 (Figs. 3B, 3C). At these stages, mOPA1 was found to be strongly expressed both in the GCL and optic fiber layer of the retina and the optic nerve (Fig. $3 \mathrm{~B}, 3 \mathrm{Bb}, 3 \mathrm{Bb}^{\prime}$ ). No expression was observed in the inner plexiform layer (IPL), inner nuclear layer (INL), outer plexiform layer (OPL), outer nuclear layer (ONL), photoreceptors (PR), or pigmented epithelium (Figs. $3 \mathrm{Aa}, 3 \mathrm{Bb}^{\prime}, 3 \mathrm{Cc}$ ). Between $\mathrm{P} 4$ and P8, there was a sharp decline in the level of mOPA1 expression in the retina, although from P4 onward, the expression spread from the GCL to the IPL and INL (Figs. 3D, 3F, 3G). This decline was concomitant with a drastic decrease in mOPA1 expression in the optic nerve (compare Figs. 3Bb and 3E). The P12 stage coincides with the time when the mouse eye opens. At this stage, a broad, diffused mOPA1 signal was detected in the GCL and the IPL, and a more 

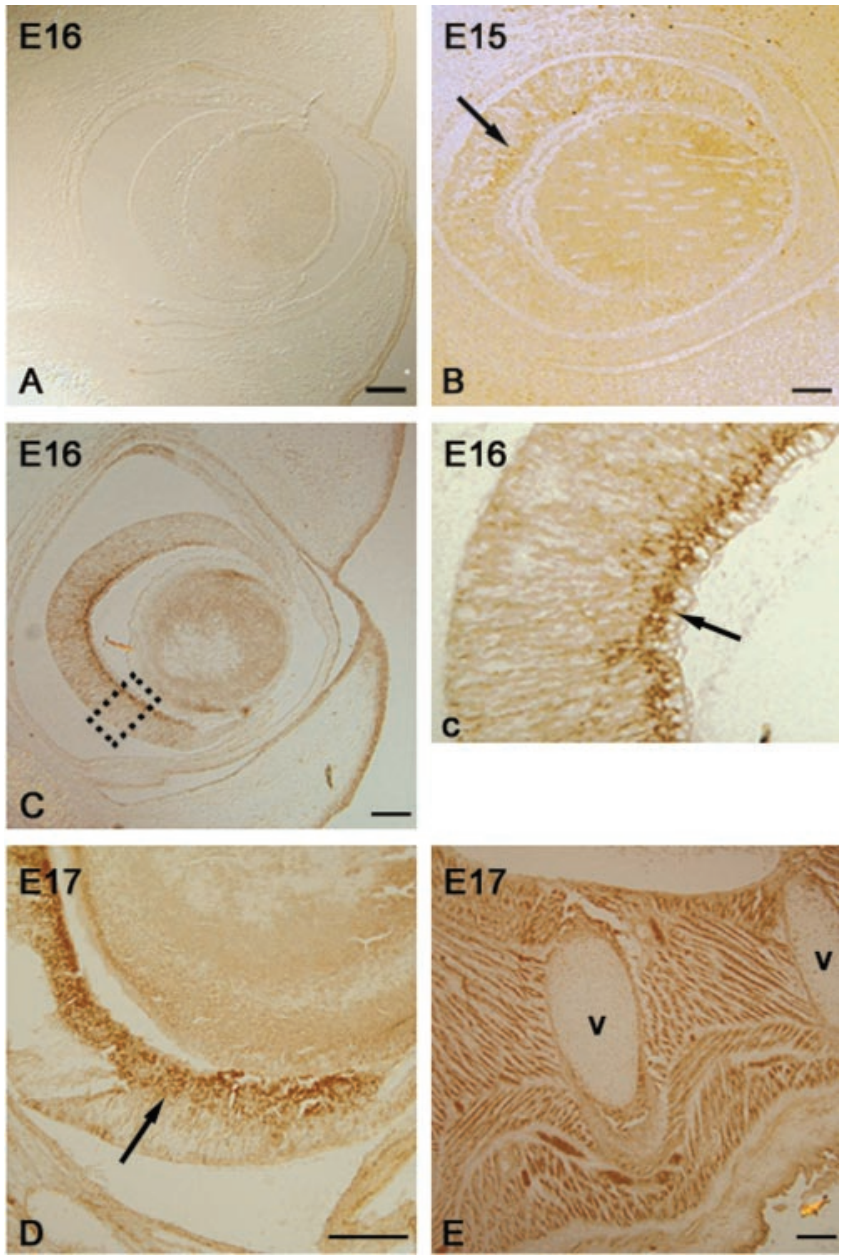

FiguRE 2. Expression profile of mOPA1 during mouse embryonic development. (A) E16 embryo stained with rabbit preimmune serum showed weak, nonspecific staining in the lens and surface ectoderm. (B) The earliest expression of mOPA1 in the retina was observed at E15, where it was present at a low level in the inner retina, corresponding to the position of the subsequent location of the GCL (B, Cc, and $\mathbf{D}$, arrows) and the lens. (C) mOPA1 was strongly expressed at E16 as a distinct band in the presumptive GCL. Weaker staining was also present in the lens and neuroblastic layer of the retina; (Cc) $40 \times$ magnification of the boxed area. (D) At E17, expression of mOPA1 was detected in the presumptive GCL of the retina. (E) At E17, mOPA1 was strongly expressed in most other body tissues. Shown is a sagittal section of the skeletal muscle around the vertebrae (v). Scale bar, 100 $\mu \mathrm{m}$.

localized signal was detected in the INL of the mouse retina (Fig. 3G). A weak signal was also observed in the myelinated region of the optic nerve (data not shown). No expression was detected in the lens or other ocular tissues at any of the postnatal stages examined (data not shown).

\section{Analysis of mOPA1 Expression in the Bst/+ Mouse Retina}

As the $B s t /+$ mouse has been proposed as a model for ADOA, ${ }^{32}$ we also examined mOPA1 expression in the adult $B s t /+$ retina and compared it with the expression pattern in the wild-type adult mouse retina. In the wild-type adult mouse retina, mOPA1 expression was detected in the GCL, IPL, INL, and OPL, although this signal appeared to be more distinctly localized in the INL than in the GCL, IPL, and OPL (Fig. 4E). Within the adult mouse optic nerve, a weak, patchy mOPA1 signal was detected in the myelinated region (Fig. 4F). No mOPA1 signal was detected in the lens or any other ocular tissue (not shown). Significantly, the weak mOPA1 expression observed in the adult mouse eye was in contrast to its strong expression in the mouse liver, which remained comparable to that at embryonic stages (data not shown). The Bst/+ mouse retina showed a similar profile of mOPA1 expression, although the spatial distribution was affected by the loss of normal structure due to rosette formation within the Bst/+ retina (Fig. 4B). It has been reported that in severely affected $B s t /+$ mice, the organization of the neural retina is distorted with the inner and outer nuclear layers thrown into folds, rosettes or colobomas and the IPL and OPL reduced in thickness. ${ }^{31}$ All these defects were clearly evident in a hematoxylin-eosin-stained section of the $B s t /+$ retina (Fig. $4 \mathrm{~A}$ ). In particular, the OPL was found to be either absent or severely reduced. Despite these structural defects, a strong mOPA1 signal was detected in the GCL and the IPL, whereas a much weaker signal was seen in the INL, surrounding the rosettes (Fig. 4C). In more structurally normal regions of the $B s t /+$ retina, mOPA1 signal was comparable to that observed in the adult wild-type mouse retina (Fig. 4D). No signal was detected in the lens or other ocular tissues of the $B s t /+$ eye (data not shown).

\section{Analysis of OPA1 Expression in the Adult Human Eye}

Figure 5 shows the expression of OPA1 in different human ocular tissues. Human OPA1 was found to be expressed not only in the GCL, IPL, and INL but also in the OPL. Notably, there appeared to be a distinct boundary of OPA1 expression at the outer limit of the OPL (Fig. 5B). No expression was detected in the ONL, PR, or the pigmented epithelium (Fig. 5B). OPA1 was also detected in the ciliary body (data not shown), the corneal epithelium and endothelium (Fig. 5C), and the optic nerve (Fig. 5D). Expression in the optic nerve was detected in the myelinated region beyond the posterior limit of the lamina cribrosa, but not in the unmyelinated prelaminar region (Fig. 5D). No reaction products were observed when the primary antibody was omitted or when the immunolabeling was performed with only rabbit preimmune serum (data not shown).

\section{Discussion}

In this study, we examined the expression profile of OPA1 in embryonic and adult mammalian tissues and compared the expression of mOPA1 between wild-type adult mouse eye and the $B s t /+$ eye. Western blot analysis of proteins from a panel of adult human tissues showed that OPA1 is ubiquitously expressed (Fig. 1), although some isoforms predominate in different tissues. The presence of OPA 1 protein in different tissues further corroborates previous work that reported OPA1 transcripts in different human tissues by Northern blot analysis of multiple tissue blots. ${ }^{16}$ Our immunohistological analyses of OPA1 expression in the adult mammalian retina revealed that it is not expressed exclusively in the GCL. In fact, we found OPA1 also to be expressed in the IPL, INL, and OPL of the adult mouse and human retina. This result is intriguing in light of the fact that ADOA is thought to be a consequence of primary degeneration of $\mathrm{RGCs}^{3}$ and no defects in the inner and OPLs or the INL have been reported in patients exhibiting ADOA. The sustained expression of OPA1 in the adult mammalian retina suggests that it may be required to perform an essential function in postnatal development.

We also determined the spatial and temporal expression pattern of mOPA1 in the developing mouse retina, which may provide fundamental clues to the in vivo function of OPA1 and 
FiguRE 3. mOPA1 expression in the mouse eye during postnatal development. (A) mOPA1 was strongly expressed in the retina at $\mathrm{PO}$ in the GCL; (Aa) $40 \times$ magnification of the boxed area in (A). The arrow indicates the RGC layer. (B) mOPA1 expression peaked at $\mathrm{P} 1$, when it was localized in the GCL and optic fiber layer of the retina and the optic nerve; (Bb) mOPA1 expression (boxed area, top) in the optic nerve at $40 \times$ magnification; ( $\mathbf{B b}^{\prime}$ ) $40 \times$ magnification of the bottom boxed area, showing mOPA1 expression in the GCL. (C) At P2, there was a marginal decline in the level of mOPA1 expression, although it was still expressed only in the GCL; (Cc) $40 \times$ magnification of the boxed area. (D) At P4, mOPA1 expression showed a sharp decline and appeared as a broad, diffuse band, not only in the GCL, but also in the IPL and INL. (E) At P4, there was also a decline in the expression of mOPA1 in the optic nerve. At P8 (F) and P12 (G), the mOPA1 expression profile was similar, being localized in the GCL, IPL, and INL.Scale bar, $100 \mu \mathrm{m}$.
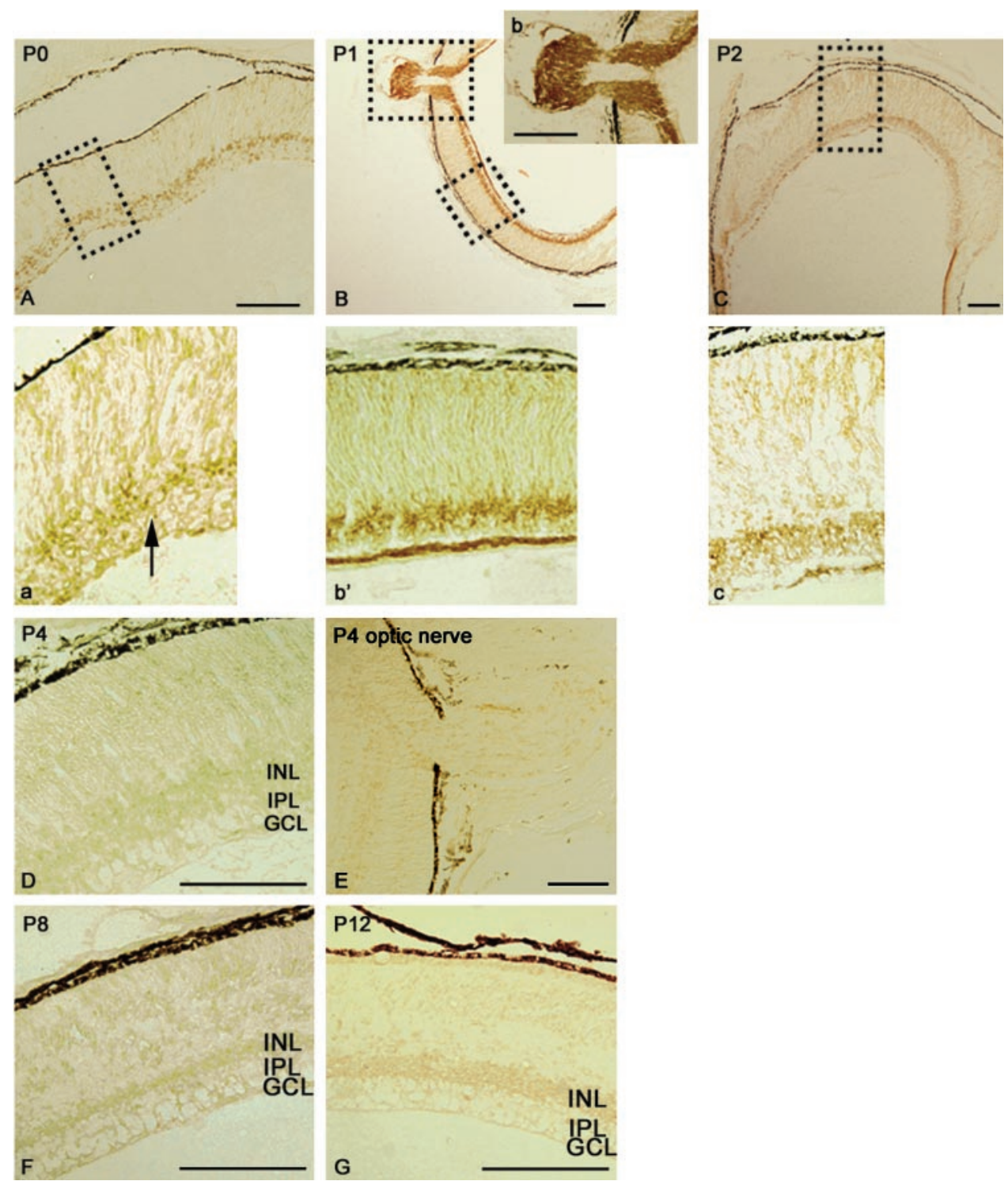

hence help our understanding of the pathogenesis of OPA1type ADOA. In our study, the earliest ocular expression of mOPA1 was detected at E15. Between E16 and E17, mOPA1 was found to be expressed very strongly in the prospective GCL of the retina and weakly in the lens (Fig. 2). No expression was detected in the developing photoreceptor layer, the pigmented epithelium, or other ocular tissues at these stages. Expression was also detected in the lung, liver, heart, skeletal muscle, and brain of the developing embryo. After birth, strong mOPA1 expression continued in the GCL of the retina and in the optic nerve until P2 and then declined sharply (Fig. 3). Between P0 and P2, mOPA1 was localized both within the GCL and the optic fiber layer of the retina and the optic nerve. Between P4 and P12, although mOPA1 levels decreased in the GCL of the retina, its expression domain was found to spread to the IPL and INL. Beyond this stage, MOPA1 was expressed at a weak level in the retina and optic nerve throughout life. However, we detected a high level of mOPA1 expression in the adult mouse liver, and Misaka et al. ${ }^{20}$ have reported high levels of $M O P A 1$ transcripts in the adult mouse brain, heart, lung, liver, kidney, ovary, and skeletal muscle. Thus, the downregulation in the mOPA1 signal that we observed in the eye in the postnatal stages is in contrast to its continued high level of expression in other body tissues. This may explain why the

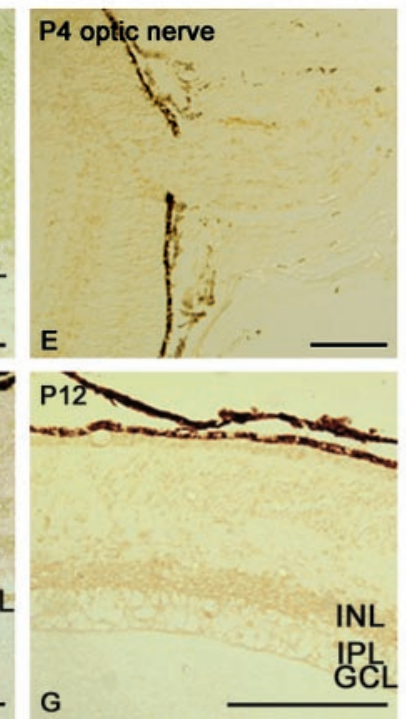

pathologic effects of ADOA are seen only in the eye, despite the ubiquitous presence of OPA1 in almost all body tissues. It may be that the sharp decline in mOPA1 expression beyond $\mathrm{P} 2$ merely reflects a natural reduction in the number of RGCs by apoptosis. ${ }^{35}$ However, the fact that OPA1 expression gradually increases before birth and peaks shortly after birth suggests another role for OPA1. In the mouse, RGCs are generated between E11 and E18, and the latter age coincides with the onset of naturally occurring RGC death. ${ }^{36}$ After E12, the RGCs extend their axons along defined paths and establish topographically ordered connections in the central nervous system. The first RGC axons reach the optic chiasm at E12 and their most distal target in the midbrain, the superior colliculus, at E14 but additional RGC axons continue to grow throughout the optic tract and into the superior colliculus until birth. ${ }^{37,38}$ By P14, the mature retinocollicular topographic map is normally established. ${ }^{37}$ The appearance of OPA1 after E14 suggests that it may play a role in RGC axon growth, pathfinding, and/or target innervation.

Patients with ADOA have a reduced number of RGCs. ${ }^{3,6} \mathrm{~A}$ similar reduced number of RGCs has also been reported in the retina of the $B s t /+$ mouse. ${ }^{31}$ Approximately $60 \%$ of $B s t /+$ mice show a reduced or complete absence of the pupillary light reflex and atrophy of the optic nerve in one or both eyes, and 

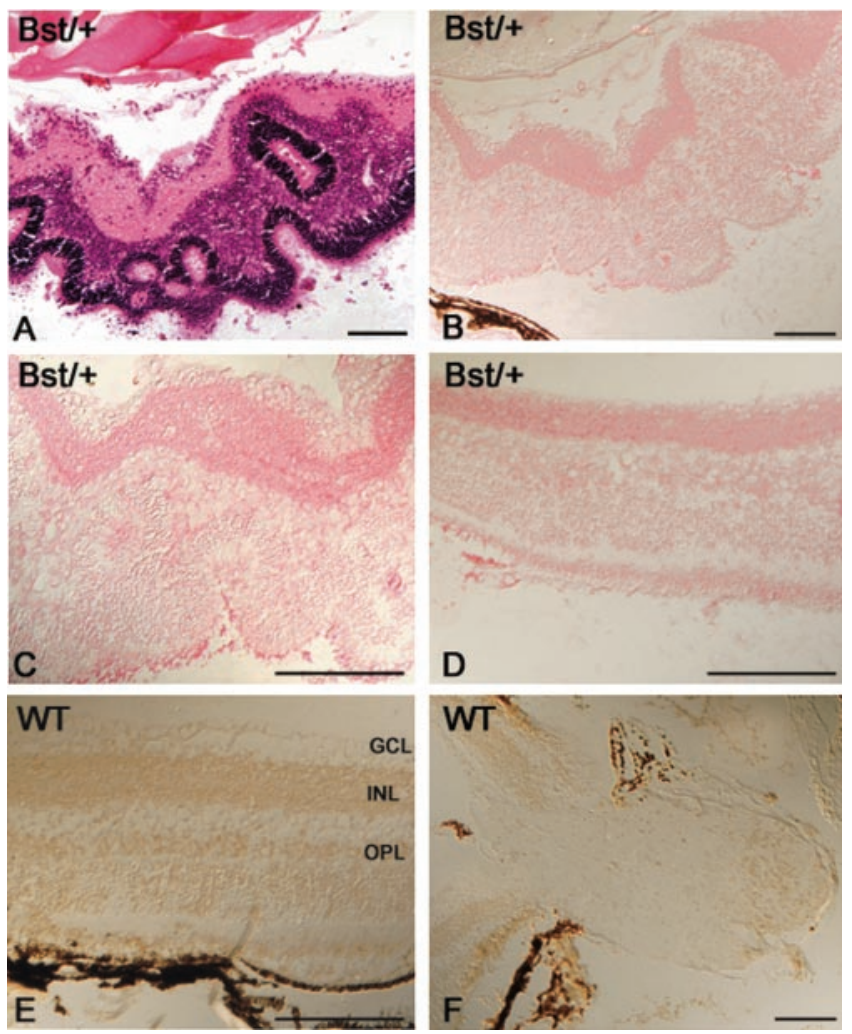

Figure 4. Comparison of mOPA1 expression in the Bst/+ and wildtype adult mouse retina. (A) Hematoxylin-eosin-stained section of the $B s t /+$ retina. (B) mOPA1 was strongly expressed in the GCL and IPL, whereas a weaker signal was observed in the INL of the Bst/+ retina in the region around the rosettes (C). (D) In a more structurally normal region, mOPA1 is strongly expressed in the GCL and IPL and weakly expressed in the INL. Immunolabeling was detected by staining with a red substrate in (B), (C), and (D). (E) In the wild-type adult mouse retina, mOPA1 was localized in the GCL, IPL, INL, and OPL. (F) In the adult mouse optic nerve, a weak mOPA1 signal was observed in the myelinated region. Immunolabeling was detected by staining with $3^{\prime}, 3^{\prime}$-diaminobenzidine in (E) and (F). WT, wild type. Scale bars, 100 $\mu \mathrm{m}$.

older mice also show subretinal neovascularization. ${ }^{31,39}$ Until recently, it was postulated that the mouse Bst locus was homologous to the human OPA1 gene as a result of a fractured synteny between the OPA1 locus at q28 on human chromosome 3 and the Bst locus on mouse chromosome $16 .^{32}$ We therefore looked at the expression of mOPA1 in the adult $B s t /+$ mouse retina and compared it with that in the wild-type adult mouse retina. Although the morphology of the Bst/+ retina was severely disrupted as a result of rosette formation, a strong mOPA1 signal was detected in the GCL and IPL, whereas a weaker signal was observed in the INL around the rosettes. Because the OPL in the Bst/+ retina was either absent or severely reduced, the signal in the OPL was unclear. In more structurally normal regions, the signal was comparable to that observed in the wild-type adult mouse retina. Therefore, the $B s t /+$ mouse may not be an accurate model for ADOA, as has been previously proposed. ${ }^{32}$ Moreover, the polydactyly observed in Bst/+ mice has never been reported in patients with ADOA, nor do the retinas of those with ADOA show the neovascularization that occurs in older Bst/+ mice. ${ }^{33,34,39}$ We do not believe the $B s t /+$ mouse to be a true model of ADOA. In fact, while this work was in progress, Delettre et al. ${ }^{40}$ excluded the mouse opa1 from the Bst locus by radiation hybrid mapping, confirming our results. Our results further underline the need to develop a mouse model of ADOA to understand the pathologic course of the disease.

Finally, in the adult human eye, we found OPA1 to be expressed in the GCL, IPL, INL, and OPL of the retina, as well as in the cornea, ciliary body, and optic nerve. Most notably, within the optic nerve, OPA1 was present in the myelinated region beyond the lamina cribrosa but not in the unmyelinated prelaminar zone. This observation is significant, because the mitochondrial marker, cytochrome-c oxidase has been shown to be relatively abundant in the unmyelinated region. ${ }^{41}$ Also, a high degree of overlap has been shown between the distribution of OPA1 and cytochrome- $c$ in transfected COS-7 cells, ${ }^{20}$ although the OPA1 signal did not overlap that of cytochrome- $c$ when primary cultures of dissociated rat cerebellar cells were used in the same study. ${ }^{20}$

Our results clearly show that at least in the adult human optic nerve, OPA1 and cytochrome- $c$ oxidase do not colocalize in vivo, suggesting that despite the homology to mitochondrial GTPases, the distribution of endogenous OPA1 may not be confined to the mitochondria. This may also explain why we do not observe any OPA1 in the PR layer, which is highly metabolically active and rich in mitochondria. In the adult mouse, weak mOPA1 expression was observed in the myelinated region of the optic nerve, and although the expression did not extend beyond the OPL in the retina, there was no distinct boundary of mOPA1 expression at the outer limit of the OPL, as seen in the human retina. These observations may reflect species-specific differences in OPA1 expression between the human and mouse eye. Thus, OPA1 may have a complex role to play in the adult mammalian eye, and more functional studies are needed to address its role in the pathogenesis of ADOA.
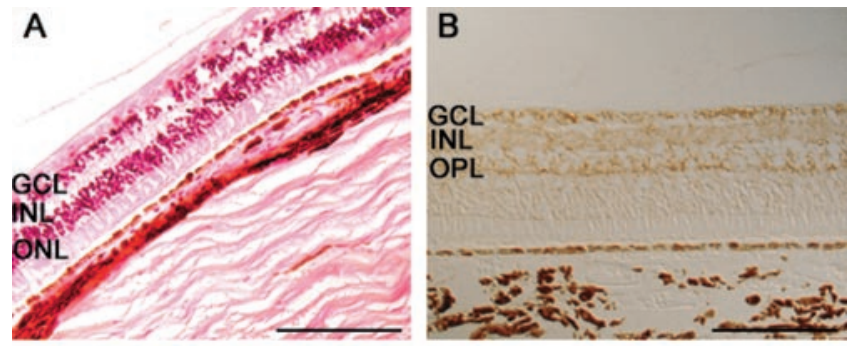

C

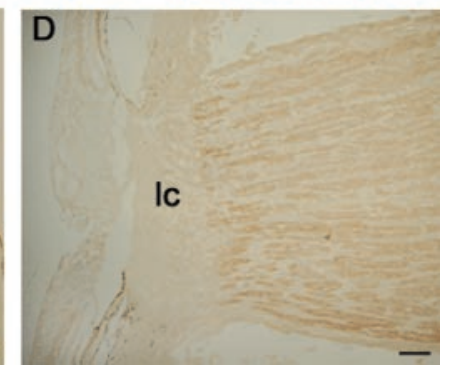

Figure 5. Expression profile of OPA1 in the adult human eye. (A) A hematoxylin-eosin-stained section of the human retina. (B) OPA1 was expressed in the GCL, IPL, INL, and OPL of the adult human retina. There is a boundary of OPA1 expression at the outer limit of the OPL. No expression is detected in the ONL or the photoreceptors. (C) OPA1 is also detected in the corneal epithelium and endothelium. (D) Strong expression of OPA1 is observed in the optic nerve where it is localized in the myelinated region beyond the lamina cribrosa, but not in the unmyelinated prelaminar zone. The gaps observed in the prelaminar region were a result of sectioning. ep, epithelium; ed, endothelium; lc, lamina cribrosa. Scale bar: (A-C) $100 \mu \mathrm{m}$; (D) $500 \mu \mathrm{m}$. 


\section{Acknowledgments}

The authors thank the Brain Bank, Department of Neuropathology, Institute of Psychiatry, London, United Kingdom, for the provision of human tissue samples.

\section{References}

1. Kjer P. Infantile optic atrophy with dominant mode of inheritance: a clinical and genetic study of 19 Danish families. Acta Ophthalmol Scand. 1959;37(suppl 54):1-146.

2. Kline LB, Glaser JS. Dominant optic atrophy: the clinical profile. Arch Opbthalmol. 1979;97:1680-1686.

3. Johnston PB, Gaster RN, Smith VC, Tripathi RC. A clinico-pathological study of autosomal dominant optic atrophy. Am J Opbthalmol. 1979;88:668-675.

4. Votruba M, Moore AT, Bhattacharya SS. Clinical features, molecular genetics and pathophysiology of dominant optic atrophy. I Med Genet. 1998;35:793-800.

5. Votruba M, Fitzke FW, Holder GE, Carter A, Bhattacharya SS, Moore AT. Clinical features in affected individuals from 21 pedigrees with dominant optic atrophy. Arch Opbthalmol. 1998;116: 351-358.

6. Kjer P, Jensen OA, Klinken L. Histopathology of eye, optic nerve and brain in a case of dominant optic atrophy. Acta Opbthalmol. 1983;61:300-312.

7. Smith DP. Diagnostic criteria in dominantly inherited juvenile optic atrophy: a report of three new families. Am J Optom Physiol Opt. 1972; 49:183-200.

8. Elenius V. Rod thresholds in dominantly inherited juvenile optic atrophy. Opbthalmologica. 1991;202:208-212.

9. Eiberg H, Kjer B, Kjer P, Rosenberg T. Dominant optic atrophy (OPA1) mapped to chromosome $3 \mathrm{q}$ region. I. Linkage analysis. Hum Mol Genet. 1994;3:977-980.

10. Kerrison JB, Arnould VJ, Ferraz Sallum JM, et al. Genetic heterogeneity of dominant optic atrophy, Kjer type: identification of a second locus on chromosome 18q12.2-12.3. Arch Opbthalmol. 1999;117:805-810

11. Anikster Y, Kleta R, Shaag A, Gahl WA, Elpeleg O. Type III 3-methylglutaconic aciduria (optic atrophy plus syndrome, or Costeff optic atrophy syndrome): identification of the OPA3 gene and its founder mutation in Iraqi Jews. Am J Hum Genet. 2001;69:12181224.

12. Assink JJ, Tijmes NT, ten Brink JB, et al. A gene for X-linked optic atrophy is closely linked to the $\mathrm{Xp} 11.4-\mathrm{Xp} 11.2$ region of the $\mathrm{X}$ chromosome. Am J Hum Genet. 1997;61:934-939.

13. Delettre C, Lenaers G, Griffoin J-M, et al. Nuclear gene OPA1, encoding a mitochondrial dynamin-related protein, is mutated in dominant optic atrophy. Nat Genet. 2000;26:207-210.

14. Votruba M, Moore AT, Bhattacharya SS. Genetic refinement of dominant optic atrophy (OPA1) locus to within a $2 \mathrm{cM}$ interval of chromosome 3q. J Med Genet. 1997;34:117-121.

15. Votruba M, Moore AT, Bhattacharya SS. Demonstration of a founder effect and fine mapping of dominant optic atrophy locus on 3q28-qter by linkage disequilibrium method: a study of 38 British Isles pedigrees. Hum Genet. 1998;102:79-86.

16. Alexander C, Votruba M, Pesch UEA, et al. OPA1, encoding a dynamin-related GTPase, is mutated in autosomal dominant optic atrophy linked to chromosome 3q28. Nat Genet. 2000;26:211215.

17. Delettre C, Griffoin JM, Kaplan J, et al. Mutation spectrum and splicing variants in the OPA1 gene. Hum Genet. 2001;109:584591.

18. Delettre C, Lenaers G, Pelloquin L, Belenguer P, Hamel CP. OPA1 (Kjer type) dominant optic atrophy: a novel mitochondrial disease. Mol Genet Metab. 2002;75:97-107.

19. van der Bliek AM. A mitochondrial division apparatus takes shape. J Cell Biol. 2000;151:F1-F4.

20. Misaka T, Miyashita T, Kubo Y. Primary structure of a dynaminrelated mouse mitochondrial GTPase and its distribution in brain, subcellular localization, and effect on mitochondrial morphology. J Biol Chem. 2002;277:15834-15842.

21. Shepard KA, Yaffe MP. The yeast dynamin-like protein, Mgm1p, functions on the mitochondrial outer membrane to mediate mitochondrial inheritance. J Cell Biol. 1999;144:711-720.

22. Wong ED, Wagner JA, Gorsich SW, McCaffery JM, Shaw JM, Nunnari J. The dynamin-related GTPase, Mgm1p, is an intermembrane space protein required for maintenance of fusion competent mitochondria. J Cell Biol. 2000;151:341-352.

23. Olichon A, Emorine LJ, Descoins E, et al. The human dynaminrelated protein OPA1 is anchored to the mitochondrial inner membrane facing the inter-membrane space. FEBS Lett. 2002;523:171176.

24. Satoh M, Hamamoto T, Seo N, Kagawa Y, Endo H. Differential sublocalization of the dynamin-related protein OPA1 isoforms in mitochondria. Biochem Biophys Res Commun. 2003;300:482493.

25. Thiselton DL, Alexander C, Taanman JW, et al. A comprehensive survey of mutations in the OPA1 gene in patients with autosomal dominant optic atrophy. Invest Ophthalmol Vis Sci. 2002;43: 1715-1724.

26. Pesch UE, Leo-Kottler B, Mayer S, et al. OPA1 mutations in patients with autosomal dominant optic atrophy and evidence for semidominant inheritance. Hum Mol Genet. 2001;10:1359-1368.

27. Toomes C, Marchbank NJ, Mackey DA, et al. Spectrum, frequency and penetrance of OPA1 mutations in dominant optic atrophy. Hum Mol Genet. 2001;10:1369-1378.

28. Thiselton DL, Alexander C, Morris A, et al. A frameshift mutation in exon 28 of the OPA1 gene explains the high prevalence of dominant optic atrophy in the Danish population: evidence for a founder effect. Hum Genet. 2001;109:498-502.

29. Kortuem K, Geiger LK, Levin LA. Differential susceptibility of retinal ganglion cells to reactive oxygen species. Invest Ophthalmol Vis Sci. 2000;41:3176-3182.

30. Southard JL, Eicher EM. Bst. Mouse News Lett. 1977;56:40.

31. Rice DS, Tang Q, Williams RW, Harris BS, Davisson MT, Goldowitz D. Decreased retinal ganglion cell number and misdirected axon growth associated with fissure defects in Bst/+ mutant mice. Invest Ophthalmol Vis Sci. 1997;38:2112-2124.

32. Rice DS, Williams RW, Ward-Bailey $P$, et al. Mapping the Bst mutation on mouse chromosome 16: a model for human optic atrophy. Mamm Genome. 1995;6:546-548.

33. Epstein R, Davisson M, Lehmann K, Akeson EC, Cohn M. Position of Igl-1, $m d$, and Bst loci on chromosome 16 of the mouse. Immunogenetics. 1986;23:78-83.

34. Tang Q, Rice DS, Goldowitz D. Disrupted retinal development in the embryonic belly spot and tail mutant mouse. Dev Biol. 1999; 207:239-255.

35. Perry VH, Henderson Z, Linden R. Postnatal changes in retinal ganglion cell and optic axon populations in the pigmented rat. J Comp Neurol. 1983;219:356-368.

36. Dräger UC. Birth dates of retinal ganglion cells giving rise to the crossed and uncrossed optic projections in the mouse. Proc $R$ Soc Lond B Biol Sci. 1985;224:57-77.

37. Simon DK, O'Leary DDM. Development of topographic order in the mammalian retinocollicular projection. J Neurosci. 1992;12: 1212-1232.

38. Marcus RC, Mason CA. The first retinal axon growth in the mouse optic chiasm: axon patterning and the cellular environment. J Neurosci. 1995;15:6389-6402.

39. Smith RS, John SWM, Zabeleta A, Davisson MT, Hawes NL, Chang B. The Bst locus on mouse chromosome 16 is associated with age-related subretinal neovascularization. Proc Nat Acad Sci USA. 2000;97:2191-2195.

40. Delettre C, Lenaers G, Belenguer P, Hamel CP. Gene structure and chromosomal localization of mouse Opa1: its exclusion from the Bst locus. BMC Genet. 2003;4:8.

41. Bristow EA, Griffiths PG, Andrews RM, Johnson MA, Turnbull DM. The distribution of mitochondrial activity in relation to optic nerve structure. Arch Opbthalmol. 2002;120:791-796. 